\title{
SRF ACCELERATOR TECHNOLOGY TRANSFER EXPERIENCE FROM THE ACHIEVEMENT OF THE SNS CRYOMODULE PRODUCTION RUN*
}

\author{
J. Hogan ${ }^{\dagger}$, T. Cannella, E. F. Daly, M. Drury, J. Fischer, T. Hiatt, P. Kneisel, J. Mammosser, \\ J. Preble, T. Whitlatch, K. Wilson, M. Wiseman \\ Thomas Jefferson Accelerator Facility, Newport News, VA 23606, U.S.A.
}

\begin{abstract}
This paper will discuss the technology transfer aspect of superconducting RF expertise as it pertains to cryomodule production, beginning with the original design requirements through testing and concluding with product delivery to the end user. The success of future industrialization of accelerator systems is dependent upon a focused effort on accelerator technology transfer. Over the past twenty years the Thomas Jefferson National Accelerator Facility (Jefferson Lab) has worked with industry to successfully design, manufacture, test and commission more superconducting RF cryomodules than any other entity in the United States. The most recent accomplishment of Jefferson Lab has been the successful production of twenty-four cryomodules designed for the Spallation Neutron Source (SNS). Jefferson Lab was chosen, by the United States Department of Energy, to provide the superconducting portion of the SNS linac due to its reputation as a primary resource for SRF expertise. The successful partnering with and development of industrial resources to support the fabrication of the superconducting RF cryomodules for SNS by Jefferson Lab will be the focus of this paper.
\end{abstract}

\section{INTRODUCTION}

The success of future superconducting radio frequency (SRF) technology based accelerators will be dependent upon effective transfer of this technology to industry. Industry must acquire the capability to efficiently manufacture SRF components, such as cryomodules, if large projects like the International Linear Collider (ILC) are to be accomplished in a reasonable time frame. Current production capacities will have to increase at least ten fold, at a minimum, in order to meet future accelerator project requirements. Jefferson $\mathrm{Lab}$ has been involved with accelerator technology transfer to industry for both research and development and project specific purposes. A core function integrated with this effort is the understanding of the interdependencies of the various systems that must work together to optimize cryomodule performance. During the SNS production run, Jefferson Lab worked with industry to fabricate the subsystems of the cryomodules while maintaining a concentrated focus on their interdependencies and the affect on performance.

\footnotetext{
*Work supported by ... US DOE Contract Nos. DE-AC05-84ER40150

†hogan@jlab.org
}

\section{CRYOMODULE SUBSYSTEMS}

The cryomodule subsystems discussed below are not meant to be completely inclusive, however they will demonstrate the critical nature of accurate technology transfer as it pertains to SRF accelerator components.

\section{Cavities}

The cavities are the most common starting point for all SRF accelerator designs and are one of the most critical components both from a performance and fabrication standpoint. The importance of the quality control associated with cavity fabrication cannot be overstated. All raw materials must be verified to ensure they meet composition and form requirements, before they are used for any part of the cavity structure. This high level of quality assurance must be maintained all the way through cavity fabrication activities to ensure the end product meets performance specifications. If the final geometry of the cavity does not meet specification, problems may develop with respect to performance (Ref Figure 1). Another concern is interfacing with tooling and mating components such as the helium vessels, which could make the part useless or result in unplanned rework costs. Knowledge of these important control points is essential to a successful fabrication activity.

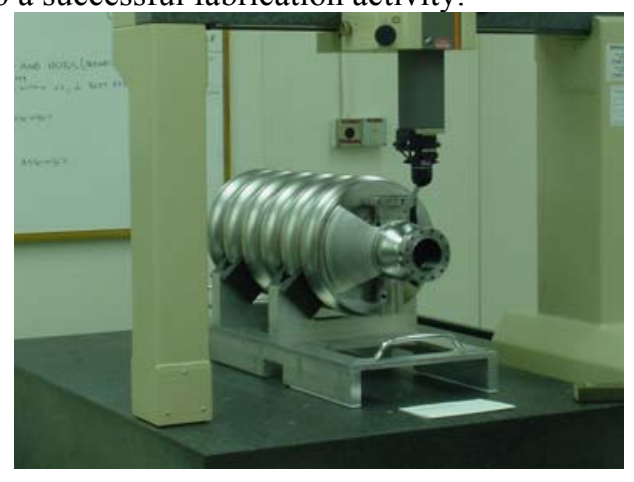

Figure 1: SNS Cavity during QA Check

\section{Power Couplers}

The technology transfer challenge associated with cavity power couplers for the SNS proved to be the manufacturability involved with the copper plating in combination with the dimensional tolerances. As is common with any new manufacturing process, acquiring a detailed understanding of the fabrication techniques and their effect on the end use is vital to providing power 
couplers that will meet or exceed all performance requirements. An important milestone of any technology transfer is reached when the recipient is able to apply the ability to identify discrepancies in their processes prior to final quality assurance inspection. The most valuable input into the development of quality assurance programs comes from documenting results of early production run activities. An additional principal aspect of the technology transfer related to the power couplers is the prompt feedback from Jefferson Lab with regard to results from their quality assurance checks. The nature of the quality assurance activities performed on these components after delivery must be well understood by industry if a reliable product is to be delivered on a routine basis.

\section{Helium Vessels}

The titanium helium vessels serve as the containment vessel for the cavities, therefore the assembly and interfacing points of these components must be precisely controlled. The critical nature of the cavity dimensional tolerances has been previously discussed with respect to this issue. The helium vessel design utilizes titanium in order to match the thermal expansion coefficient of the niobium cavities. Manufacturing titanium helium pressure vessels is not as common in industry as compared to materials such as carbon or stainless steel. One significant challenge related to titanium helium vessel fabrication is the welding process. Titanium can become embrittled if the welding is performed improperly. Industry understands these challenges associated with titanium fabrication. The critical part of technology transfer involved with the helium vessels, is the understanding of the subsequent mating requirements and operation of these components. Additionally, quality assurance procedures such as LN2 cold shocking and helium leak checking are not commonly practiced procedures outside the cryogenic industry. When working towards the industrialization of SRF accelerator technology, the transfer of that technology must be focused on a thorough understanding of the systems involved in the processes.

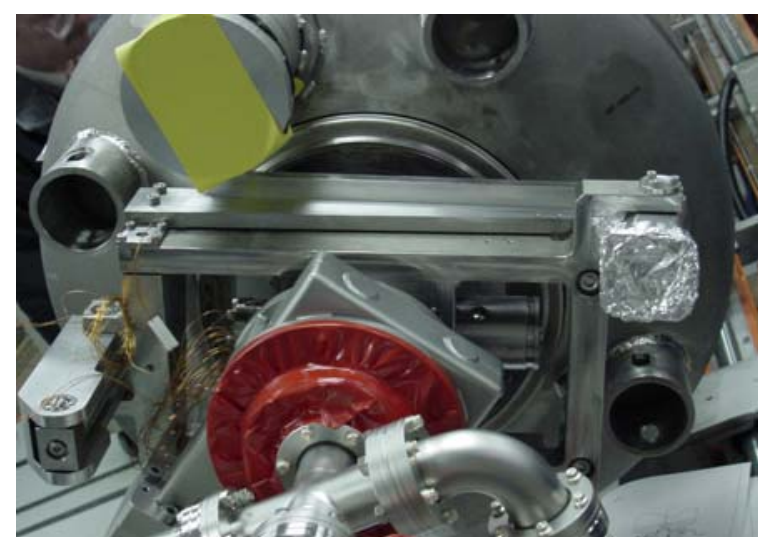

Figure 2: Cavity, Helium Vessel \& Tuner

\section{Cavity Frequency Tuners}

The cavity frequency tuner design and fabrication must accommodate mechanical interfacing with both the cavity and the helium vessel. The operations of these three components are interdependent upon each other (Reference Figure 2). These interdependencies include mechanical connections, thermal transitions, and repetitive cold operations. The moving parts necessary for proper tuner operation present opportunities for system failure. Therefore, the tuner mechanism must be manufactured with an understanding and appreciation for these control points and operational characteristics. The ability for any part of this mechanism to become bound or loose may result in system failure and for that reason must be eliminated During the SNS production run, Jefferson Lab worked with local industry in order to transfer this manufacturing knowledge, which resulted in the successful production of all the assemblies currently installed and operating in the SNS cryomodules.

\section{Integral Mechanical Components}

The two industrial sheet metal related manufacturing activities associated with SRF cryomodule technology are the magnetic and thermal shielding components. While these components may appear to be mundane at first glance, the industrial knowledge needed to manufacture these components is not trivial. These components must function appropriately both at room and cryogenic temperatures. Jefferson Lab has worked with industry over the years to develop new resources to provide these services. Other, more substantial mechanical components provided by industry include the spaceframe and vacuum vessel. Again Jefferson Lab has worked with industry to transfer the technology needed to accurately and efficiently manufacture these valuable SRF accelerator components. As previously stated, the focus of this technology transfer effort is to provide industry with the ability to understand the effect processing steps may have on the end use of the parts being manufactured. For the SNS project, Jefferson Lab was able to work with industry very closely to ensure the fabrication techniques being practiced did not impinge upon the end use of the cryomodule components. As projects become larger, it will no longer be efficient to follow up with industry partners so closely. Therefore the knowledge associated with the design and functionality of SRF accelerator components must be acquired by industry.

\section{Documentation}

Documentation has been an undertone throughout this report. In order for any technology transfer endeavor to be considered a success, documentation of the requirements, specifications, deliverables and objective must be clearly stated and understood by all participants. This condition for accomplishment is equally important for both the transmitter of the technology and for the recipient. Documentation detailing the interdependencies involved within cryomodule construction and operation 
will benefit both the physics and industrial communities. The SNS cryomodule production run at Jefferson Lab has resulted in numerous papers being published and procedures being developed. Each cryomodule delivered to SNS was accompanied with a documentation file that included drawings, specifications, quality assurance examination results, assembly procedures and component tracking information.

\section{ASSEMBLY TECHNOLOGY}

The next phase of technology transfer associated with SRF accelerator cryomodule systems is the framework of cryomodule assembly. It is during the assembly portion of the cryomodule construction where the understanding of component operational interdependencies needs to be most fundamental. The final assembly of the cryomodule components is the last opportunity to address any operational concerns or problems with individual component manufacturing. Mechanical connections are obviously one of the most evident discrepancies when present, however, assembly personnel must also be knowledgeable regarding less obvious concerns. For example, a thermal short in the helium vessel support structure could be detrimental to cryomodule performance; therefore this condition must be recognizable to assembly personnel. This knowledge must be applied routinely throughout the assembly process to ensure optimal performance.

\section{Continued Partnering}

Jefferson Lab continues to partner with industry in the field of SRF accelerator technology transfer. During the SNS cryomodule production run, Jefferson Lab partnered with industry to procure all the major cryomodule components and managed the final assembly of these components with in-house resources. Currently Jefferson
Lab is working with industry to transfer this next stage of cryomodule fabrication technology. Many of these required skills are present in industry, nonetheless, all of the skills needed with respect to the fabrication knowledge and techniques associated with cryomodule construction are not commonly found in one place in industry today. Jefferson Lab is working to change this fact by establishing working relationships within industry in order to develop the resources needed for future accelerator projects. A sample of the skills and techniques matrix in reference include alignment, helium leak checking, welding, vacuum component assembly, clean room procedures, circuit wiring, electrical signal troubleshooting and tooling applications.

\section{SUMMARY}

Jefferson Lab has been working with industry in the SRF accelerator field for many years in a conscious effort to develop these resources in order to provide the services needed by the accelerator community. The technology associated with cryomodule design and construction has advanced considerably over the past two decades and promises to advance further in the future. Jefferson Lab has been and will continue to be a strong advocate for the promotion of SRF accelerating technology. With the advent of new and larger accelerator projects, such as the International Linear Collider project, the need to rely on industry to provide SRF accelerator components is becoming imperative. With this goal in mind, Jefferson Lab will continue to share its expertise in this area with industry in order to develop a strong industrial SRF accelerator base. 